\section{NO-tryptophan: a new small molecule located in the rat brain}

\author{
A. Mangas, ${ }^{1,2,3}$ J. Yajeya, ${ }^{4}$ N. González, ${ }^{1,2}$ \\ S. Duleu, ${ }^{2}$ M. Geffard, ${ }^{1,2}$ R. Coveñas ${ }^{3}$ \\ 1 Gemacbio, Saint-Jean-d'Illac, France \\ Institute for the Development of \\ Research in Human Pathology and \\ Therapeutic (IDRPHT), Talence, France \\ ${ }^{3}$ Institute of Neurosciences of Castilla y \\ León (INCYL), Laboratory 14, University \\ of Salamanca, Spain \\ ${ }^{4}$ School of Medicine, Department of \\ Physiology, University of Salamanca, \\ Spain
}

\section{Abstract}

A highly specific monoclonal antibody directed against nitric oxide-tryptophan (NOW) with good affinity $\left(10^{-9} \mathrm{M}\right)$ and specificity was developed. In the rat brain, using an indirect immunoperoxidase technique, cell bodies containing $\mathrm{NO}-\mathrm{W}$ were exclusively found in the intermediate and dorsal parts of the lateral septal nucleus. No immunoreactive fibres were found in the rat brain. This work reports the first visualization and the morphological characteristics of cell bodies containing NO-W in the mammalian brain. The restricted distribution of NO-W in the rat brain suggests that this molecule could be involved in specific physiological mechanisms.

\section{Introduction}

Nitric oxide (NO) plays an important role in inflammatory and neurodegenerative disease $^{1-3}$ and, in this sense, the possible involvement of this molecule in the pathogenesis of multiple sclerosis has been suggested. ${ }^{4-6}$ It is known that NO modifies amino acids (e.g., tryptophan, cysteine, histidine) originating NO-amino acid species which generate an immune response. ${ }^{7,8}$ Thus, after an immunoscreening, the presence of NO-tryptophan (NO-W) by ELISA techniques has been reported in the sera of patients suffering from multiple sclerosis and in experimental animal models (e.g., experimental allergic encephalomyelitis) by the identification of circulating antibodies directed against NO-W. ${ }^{8,9}$ The detection of these antibodies show indirectly the presence of NO-W. ${ }^{8,9}$ However, to date, no study has been carried out regarding the distribution of fibres and cell bodies containing NO-W in the mammalian central nervous system. The role of this $\mathrm{NO}$ molecule remains to be studied in-depth and hence it is important to know its neuroanatomical distribution. In light of the above, the first aim of this study was to raise a highly specific monoclonal antibody directed against NO-W and the second to know the distribution of immunoreactive structures containing $\mathrm{NO}-\mathrm{W}$ in the rat brain by using an immunohistochemical method.

\section{Materials and Methods}

The experimental design, protocols, and procedures of this work were performed under the guidelines of the ethics and legal recommendations of Spanish, French and European law. This work was also approved by the experimental research commission of the University of Salamanca (Spain).

A primary antiserum was developed in BALB/c mice (Janvier Labs, Le Genest-SaintIsle, France) after immunization with NO-Wbovine serum albumin (BSA) immunogen linked via glutaraldehyde (G) [NO-W-G-BSA (NO-W conjugate)]. G complexes were prepared as previously described; ${ }^{10}$ thus, $10 \mathrm{~mL}$ of $\mathrm{W}$ were dissolved in $1 \mathrm{~mL}$ of $1.5 \mathrm{M}$ acetate buffer ( $\mathrm{pH} 8.0$ ) and a second solution was prepared containing $20 \mathrm{mg}$ of BSA dissolved in $1 \mathrm{~mL}$ of the same buffer. In order to conjugate W with BSA, $100 \mu \mathrm{L}$ of a $2.5 \mathrm{M}$ G-water solution were added to the solution containing the $\mathrm{W}$ dissolved; at this step, the first and the second solutions were mixed at room temperature, reduced and dialyzed as previously described..$^{10}$ The final dialyzed solution was nitrosylated by acidifying the antigen solution with $2 \mathrm{~N} \mathrm{HCl}$ ( $75 \mu \mathrm{L}$ per mg of a $1 \mathrm{mg} / \mathrm{mL} \mathrm{W-G-BSA} \mathrm{solution),}$ mixed with $100 \mu \mathrm{L}$ of an aqueous sodium nitrite solution (100 $\mu \mathrm{L}$ per mg of W-G-BSA) and incubated for $2 \mathrm{~h}$ shaking at $37^{\circ} \mathrm{C}$. At the end, the nitrosylated conjugate was purified in phosphate buffered saline (PBS) using dialyze membranes with cut-off limits between 12 and $16 \mathrm{KDa}$. The purification was carried out in a 1 litre bucket at $4^{\circ} \mathrm{C}$ for $2 \mathrm{~h}$ and the nitrosylation was controlled by ultraviolet spectroscopy, scanning the conjugate solution between 250 to $500 \mathrm{~nm}$. The characteristic peak at $336 \mathrm{~nm}$ confirmed the correct nitrosylation of the W-GBSA conjugate. Moreover, an indirect ELISA test, using a rabbit polyclonal antibody directed against NO-W-G-BSA (AP071; Gemacbio, Saint-Jean-d'Illac, France), was carried out and confirmed that the antigen was specifically recognized by this polyclonal antibody.

After the synthesis of NO-W-G-BSA, mice were immediately immunized by one injection every 2 or 3 weeks with the immunogen (containing NO-W-G-BSA). Each immunization
Correspondence: Dr. Arturo Mangas, Institute of Neurosciences of Castilla y León (INCYL), Laboratory of Neuroanatomy of the Peptidergic Systems (Lab. 14), University of Salamanca, c/ Pintor Fernando Gallego 1, 37007 Salamanca, Spain.

Tel. +34.923 .294500 ext. 5315

Fax: +34.923.294549.

E-mail: mangasam@usal.es

Key words: Lateral septal nucleus; septum; monoclonal antibody; cell body; nitric oxide; immunohistochemistry.

Funding: this work has been supported by the European Union FP7 Collaborative Grant TargetBraIn (279017), GEMAC S.A. Laboratories (Saint-Jean-d'lllac, France), and by the IDRPHT (Talence, France).

Received for publication: 30 May 2016.

Accepted for publication: 24 August 2016.

This work is licensed under a Creative Commons Attribution-NonCommercial 4.0 International License (CC BY-NC 4.0).

(C) Copyright A. Mangas et al., 2016

Licensee PAGEPress, Italy

European Journal of Histochemistry 2016; 60:2692 doi:10.4081/ejh.2016.2692

(subcutaneous injection) was carried out by administering $50 \mu \mathrm{L}$ of an immunogenic $\mathrm{NaCl}$ solution and $50 \mu \mathrm{L}$ of complete (only used in the first immunization) or incomplete Freund adjuvant (Sigma Aldrich, St. Louis, MO, USA). As previously described, ${ }^{10,11}$ after the second immunization serum samples were collected and the antisera were pre-purified by immunoabsorption and tested by ELISA. Once a highly specific polyclonal antibody was obtained, the fusion of SP2/0/Ag myeloma cells and mice splenocytes was carried out. Then, the screening and the selection of specific clones were conducted. The screening was carried out in order to select the best well-plate containing the cell that produces a specific monoclonal antibody. Once the highly specific monoclonal antibody directed against the NOW conjugate was obtained, cells were expanded in plastic flasks. Supernatant was recuperated every week, centrifuged and pre-purified with a saturated $\left(\mathrm{NH}_{4}\right)_{2} \mathrm{SO}_{4}$ solution, dialyzed in PBS and finally purified in an HiTrap protein G HP column (17-0404-01, GE Healthcare. Little Chalfont, UK). Later, an Isotyping kit (26179, ThermoScientific, Waltham, MA, USA) was used to determinate the type of immunoglobulin and chain. Thus, the antibody used here was characterized as an isotype Ig $\mathrm{G}_{2 \mathrm{~b}}$ with a kappa chain.

Five adult male Wistar rats (weight 300 $350 \mathrm{~g})$ obtained from commercial sources 
(Charles River, Écully, France) were used in this study. Animals were kept under standardized conditions, anaesthetized, heparinized and perfused via the ascending aorta with cold physiologic saline and with a solution containing $4 \%$ paraformaldehyde and $2 \%$ of glutaraldehyde. ${ }^{10,12}$ As previously reported, ${ }^{10,12}$ brains were dissected out, post-fixed in $4 \%$ paraformaldehyde for 12-16 h and cryoprotected for histological studies. Using a freezing microtome, 40-50 $\mu$-thick brain sections were obtained, kept in PBS solution (0.1 M, pH 7.2) and processed free-floating for immunohistochemistry. ${ }^{10,12}$ Thus, in order to avoid possible interference by endogenous peroxidase, sections were treated with methanol and $\mathrm{H}_{2} \mathrm{O}_{2}$ (2:1) for $30 \mathrm{~min}$, washed in PBS and pre-incubated in PBS containing $0.3 \%$ of Triton X-100 and $1 \%$ of normal horse serum (mix solution) for $30 \mathrm{~min}$. Sections were incubated overnight at $4^{\circ} \mathrm{C}$ in the mix solution containing the monoclonal anti-NO-W antiserum (diluted $1 / 1,000)$, washed in PBS pincubated with biotinylated anti-mouse immunogammaglobulin (BA-9200, Vector Labs, Burlingame, CA, USA), diluted $1 / 200$ in the mix solution (60 min). After a rinse in PBS, sections were incubated with the avidin-biotin-peroxidase complex (Vectastain PK-6100, Vector, USA) (1/100) for $60 \mathrm{~min}$. Sections were washed in PBS (30 $\mathrm{min})$ and in Tris-HCl buffer (10 min) and then the tissue-bound peroxidase was developed with $\mathrm{H}_{2} \mathrm{O}_{2}$, using 3,3 ' diaminobenzidine as chromogen. Finally, the sections were washed in PBS and coverslipped with PBS/Glycerol (1:3). Histological controls were carried out to confirm the specificity of the immunoreactivity: 1) omission of the primary and/or secondary antisera; and 2) pre-absorption of the antiNO-W antibody with an excess (100 g/mL) of NO-W. No residual immunoreactivity was found in either case. The stereotaxic atlas of Paxinos and Watson ${ }^{13}$ was used for mapping. The size and density of cell bodies were considered as previously described. ${ }^{14}$ The density was considered high (more than 20 perikarya/nucleus/section were visualized), moderate (10-20 perikarya/nucleus/section) or low (less than 10 perikarya/nucleus/section). Cell bodies were small (showing a diameter below $15 \mu \mathrm{m}$ ), medium-sized (diameter between 15-25 $\mu \mathrm{m}$ ) or large (diameter above $25 \mu \mathrm{m})$. Photomicrographs were obtained with an Olympus DP50 digital camera attached to a Kyowa Unilux-12 microscope. To improve the visualization of results, only the brightness and contrast of the images were adjusted, without any further manipulation of the photographs. Adobe Photoshop CS software was used to view the images and modify their brightness and contrast.

\section{Results}

Using ELISA tests, the monoclonal antibody obtained was fully characterized (Table 1). In these assays, the parameters studied were titration, avidity, and specificity versus other structural analogues (Table 1; Figure 1) according to protocols previously described..$^{10,12}$ ELISA tests were carried out with the dilution of $1 / 75,000$, corresponding to an optical density of 1, at $492 \mathrm{~nm} \cdot .^{10,12,15}$ Accordingly, competition experiments were performed with different competitors (Table 1). All competition experiments (dilution: 1/75,000) were carried out with the same amount of antibody but using different concentrations of the conjugated

Table 1. Affinity and specificity of antibodies directed against conjugated NO-tryptophan.

\begin{tabular}{lc} 
Compound & Cross reactivityat half-displacement (IC50) \\
NO-Tryptophan-Gc & 1 \\
L-Tryptophan-Gc & $1 / 100$ \\
\hline NO-Tyrosine-Gc & $1 / 50$ \\
NO-Cysteine-Gc & $1 / 50,000$ \\
\hline NO-Histidine-Gc & $1 / 50,000$ \\
\hline
\end{tabular}

Using competition ELISA assays, cross-reactivity was calculated from the displacement curves at half-displacement (see Figure 1): the best recognized conjugate was NO-Tryptophan-Gc, whose concentration was divided by the concentration of each of the other conjugates. Gc, glutaraldehyde protein carrier.

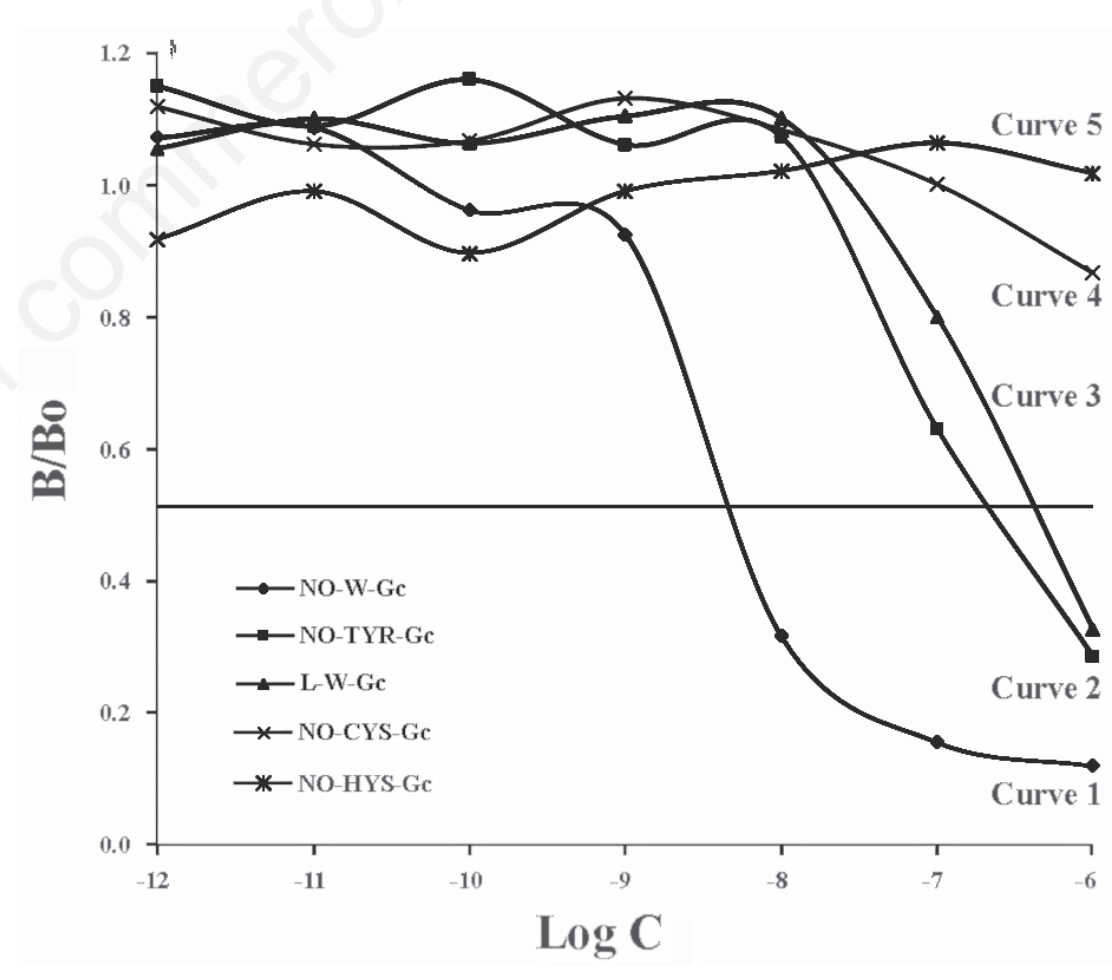

Figure 1. Antibody affinity and specificity resulting of competition experiments in ELISA test. B, optical density with competitor; B0, optical density without competitor. Curve 1, competition with NO-W-Gc; curve 2, competition with NO-Tyrosine (TYR)-Gc; curve 3, competition with L. W-Gc; curve 4, competition with NO-Cysteine (CYS)-Gc; curve 5, competition with NO-Histidine (HYS)-Gc; Gc, conjugated via glutaraldehyde to the protein carrier; Log C, logarithm of concentration. 
mm. A high density of NO-W-immunoreactive perikarya was observed by the whole rostrocaudal extension of the intermediate part of the lateral septal nucleus (Figure $2 \mathrm{C}$-F) and a low density by the whole rostro-caudal extension of the dorsal part of the lateral septal nucleus (Figure 2C). No immunoreactive cell body was observed in the ventral part of the lateral septum. In general, immunoreactive cell bodies were round or piriform and small in size. One-two dendrites were also observed (Figure $2 \mathrm{C}-\mathrm{F}$ ). The immunoreactivity was not observed when the anti-NO-W antibody was pre-absorbed with NO-W (Figure 2B). clonal antibody used here was placed in the position $\mathrm{N}$ of the NO-W. NO is a very sensible species which is very quickly oxidized to $\mathrm{NO}_{2}$; this must be taken into account during the different phases of the production of antibodies (polyclonal and monoclonal) and when testing samples and conducting the screening. Thus, as reported above, in each step of the process a new antigen was produced and immediately controlled. In this sense, the pre-absorption of the monoclonal antibody used in this work was carried out using a new and recently synthesized NO-W conjugate.
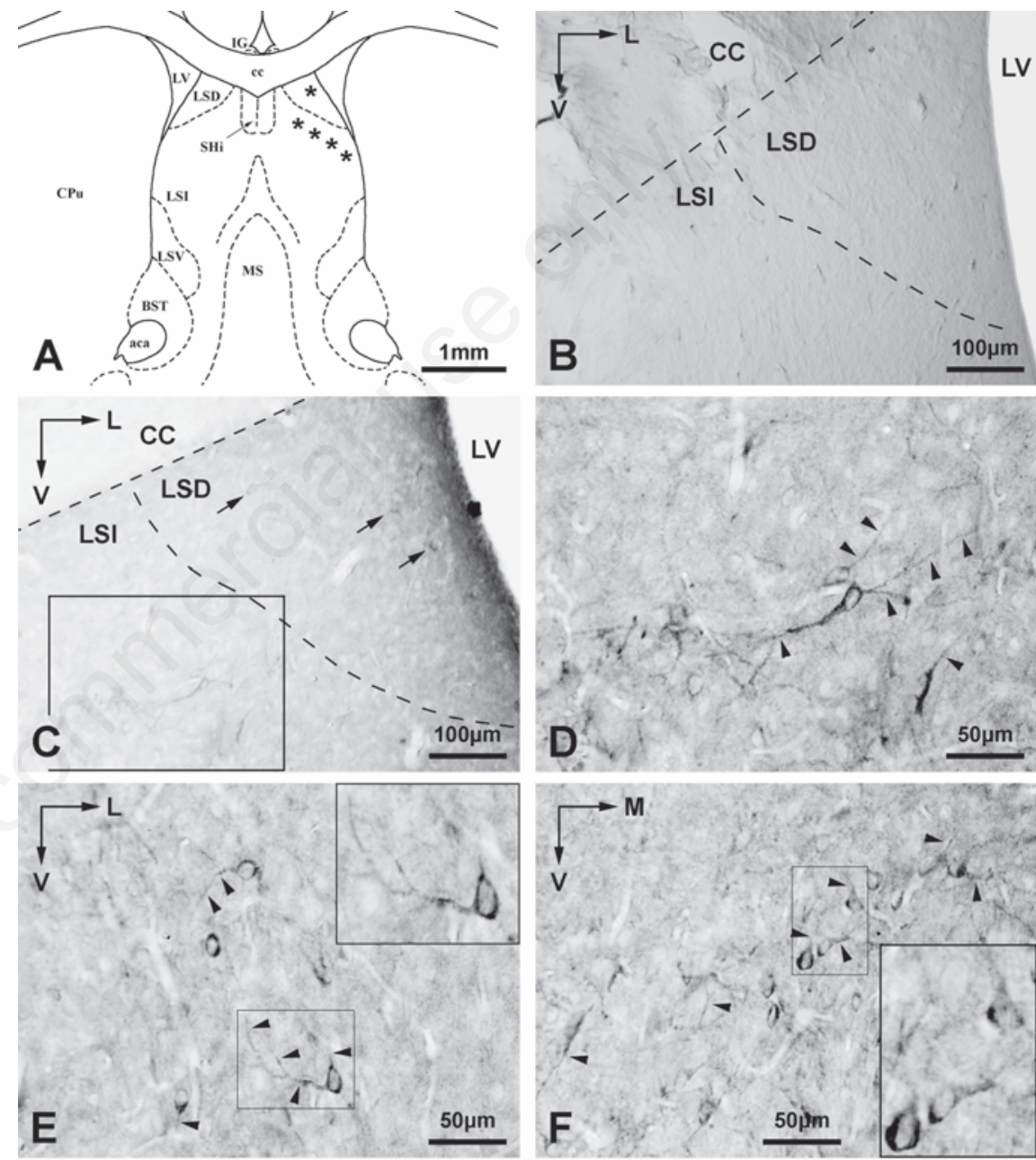
specifically due to NO-W. This is in agreement with the restricted distribution of the immunoreactivity observed (exclusively found in one nucleus of the rat brain). Moreover, this distribution and the degree of the immunoreactivity were similar in all the animals studied.

Regarding the development of antibodies directed against NO-W, it is important to remark that before the fusion of the splenocyte with the myeloma cell, a polyclonal antibody (directed against NO-W) showing a very high affinity and specificity was developed. Moreover, the purification of the antibody is a crucial step when developing new monoclonal antibodies. Here, the monoclonal antibody used was pre-purified and purified using protein G HP columns. After using an Isotype kit, the finally characterized antibody was determined: isotype $\operatorname{Ig} G_{2 b}$ with a kappa chain. Then, after the fusion, a screening was conducted in order to select the best cell producing a specific monoclonal antibody. This highly specific antibody was characterized by competition experiments and allowed to discriminate NO$\mathrm{W}$ from the closest molecules. We found that NO-W was, at least 100 times, better recognized than the conjugated $\mathrm{W}$. This highly specific target discrimination is similar to that previously shown for antibodies discriminating $\mathrm{L}$ and $\mathrm{D}$ isomers of the same aminoacid, ${ }^{10}$ since the only difference between $\mathrm{NO}-\mathrm{W}$ and $\mathrm{W}$ is due to the NO group. Moreover, it is important to note that during the nitrosylation of $\mathrm{W}$ there are two possible nitrosylation positions: $\mathrm{N}$ and $\mathrm{C}_{6}$. $\mathrm{N}$ position is preferentially nitrosylated when comparing with $\mathrm{C}_{6}$ position. This means that the NO-W recognized by the mono-
This work describes for the first time the tion of NO-W-immunoreactive cell bodies in the mammalian brain. By using the Golgi method, different morphological types of neurons have been found in the three parts of the rat lateral septal nucleus. ${ }^{16}$ Our findings are in agreement with this previous study, ${ }^{16}$ since in some cases, the morphological characteristics of the NO-W-immunoreactive neurons (round or piriform cell bodies showing two dendrites) are similar to those neurons reported in the presence and the morphological characterizadorsal and intermediate parts of the lateral
Figure 2. A) Frontal section (Bregma $0.2 \mathrm{~mm}$ ) of the rat telencephalon. NO-W-immunoreactive cell bodies (asterisks) in the dorsal (LSD) and intermediate (LSI) parts of the lateral septal nucleus. B) Note the absence of immunoreactivity in both the intermediate (LSI) and dorsal (LSD) parts of the lateral septal nucleus after the pre-absorption of the first antibody; compare this with panel C. C) Cell bodies (arrows) containing NO-W located in the intermediate (LSI) and dorsal (LSD) parts of the lateral septal nucleus. D) Higher-power magnification of the region delimited by the rectangle shown in panel C. E,F) NO-W-immunoreactive perikarya located in the intermediate part of the lateral septal nucleus; arrowheads indicate dendrites; inset: higher-power magnification of the region delimited by the rectangle. Aca, anterior commissure, anterior part; BST, bed nucleus of the stria terminalis; cc, corpus callosum; CPu, caudate-putamen; D, dorsal; IG, induseum griseum; L, lateral; LSD, lateral septal nucleus, dorsal part; LSI, lateral septal nucleus, intermediate part; LSV, lateral septal nucleus, ventral part; LV, lateral ventricle; MS, medial septal nucleus; Shi, septohippocampal nucleus; V, ventral; VDBD, nucleus of the vertical limb of the diagonal band, dorsal part. 
septal nucleus after using the Golgi method. ${ }^{16}$ It is known that the different parts of the lateral septum (ventral, intermediate, dorsal) are interconnected. ${ }^{17}$ However, according to our morphological observations, no immunoreactive fibre has been observed in the rat brain. The absence of NO-W in fibres could be due to the intraneuronal transport mechanisms of this molecule (stored exclusively in perikarya and dendrites), as it has been reported for some neuropeptides and D-amino acids (e.g., somatostatin, D-glutamate)..$^{10,18}$ It is also possible that in fibres the level of NO-W is very low and then the immunocytochemical technique is not sensitive enough to detect it.

Currently, the physiological actions of NO-W located in the mammalian brain are unknown. However, the presence of NO-W in the lateral septal nucleus suggests that this molecule could be involved in affective and motivational processes. ${ }^{17}$ The lateral septum receives inputs from the hippocampus, hypothalamus, midbrain periaqueductal gray, ventral tegmental area, locus ceruleus, amygdala and the entorhinal cortex, whereas it sends projections to regions of the central nervous system (e.g., diencephalon, limbic system) that control affective and motivational processes..$^{17,19-21}$ It is known that the lateral septum integrates cognitive and affective information and then transmits it to regions of the central nervous system that control the behavioural response. ${ }^{17}$

The data reported here are important because for the first time the presence of NO$\mathrm{W}$ is reported in normal animals. It is known that $\mathrm{NO}$ is produced during the course of several pathologies (e.g., multiple sclerosis) and that this molecule is involved in the modification of specific and different targets (e.g., NO$\mathrm{W}$, N0-cysteine, N0-tyrosine). ${ }^{8,9} \mathrm{NO}$ is an unstable molecule hard to quantify in vivo and currently the local production of NO in tissues remains technically difficult to detect. However, the characterization of circulating antibodies directed against NO-modified selfantigens (e.g., anti-NO-W) provides indirect evidence for the involvement of NO in pathogenic processes. ${ }^{8,9}$ Moreover, it has been suggested that NO is involved in the blood-brain barrier breakdown and that this molecule participates in inflammatory processes. ${ }^{22}$

In conclusion, we demonstrate here for the first time the presence of immunoreactive perikarya containing NO-W in the mammalian central nervous system after using a highly specific monoclonal antibody against NO-W. The distribution of these cell bodies is quite restricted. Our study will serve in the future to compare the results reported here in normal animals with those reported in experimental animal models showing different pathologies (e.g., multiple sclerosis, ischemia, depression). For example, in multiple sclerosis the involvement of $\mathrm{NO}$ in the pathogenesis of the disease has been suggested ${ }^{4-6}$ and hence it should be important to know whether the distribution of NO-W-immunoreactive structures is widespread or restricted in the human central nervous system. Moreover, it is known that the mammalian lateral septum is involved in mood, social behaviour, fear, depression and motivation. ${ }^{17}$ In the future, our neuroanatomical study will serve for a better understanding of the physiological roles played by the NO-W in the mammalian lateral septum.

\section{References}

1. Dawson TM, Dawson VL. ADP-ribosylation as a mechanism for the action of nitric oxide in the nervous system. New Horiz 1995;3:85-92.

2. Bolaños JP, Almeida A, Stewart V, Peuchen S, Land JM, Clark JB, et al. Nitric oxidemediated mitochondrial damage in the brain: mechanisms and implications for neurodegenerative diseases. J Neurochem 1997;68:2227-40.

3. Parkinson JF, Mitrovic B, Merrill JE. The role of nitric oxide in multiple sclerosis. $\mathrm{J}$ Mol Med 1997;75:174-86.

4. Bo L, Dawson TM, Wesselingh S, Mork S, Choi S, Kong PA, et al. Induction of nitric oxide synthase in demyelinating regions of multiple sclerosis brain. Ann Neurol 1994;36:778-86.

5. Hooper DC, Bagasra 0, Marini JC, Zborek A, Ohnishi ST, Kean R, et al. Prevention of experimental allergic encephalomyelitis by targeting nitric oxide and peroxynitrite: implications for the treatment of multiple sclerosis. Proc Natl Acad Sci USA 1997; 94:2528-33.

6. Jolivalt CG, Howard RB, Chen LS, Mizisin AP, Lai CS. A novel nitric oxide scavenger in combination with cyclosporine A ameliorates experimental autoimmune encephalomyelitis progression in mice. $\mathrm{J}$ Neuroimmunol 2003;138:56-64.

7. Boullerne AI, Petry KG, Meynard M, Geffard M. Indirect evidence for nitric oxide involvement in multiple sclerosis by characterization of circulating antibodies directed against conjugated S-nitrocysteine. J Neuroimmunol 1995;60:117-24.

8. Bodet D, Mangas A, Coveñas R, Dabadie MP, Geffard M. Indirect visualization of specific antigenic modifications induced by nitric oxide in an experimental model of allergic encephalomyelitis. In: M. Geffard (ed.) Multiple sclerosis: a new paradigm. New York: Nova Science Publishers; 2011. p. 133-52.

9. Bodet D, Glaize G, Dabadie MP, Geffard M.
[Suivi immunobiologique de malades atteints de sclérose en plaques].[Article in French]. Immunoanal Biol Spéc 2004;19:138-47.

10. Mangas A, Coveñas R, Bodet D, Geffard M, Aguilar LA, Yajeya J. Immunocytochemical visualization of D-glutamate in the rat brain. Neuroscience 2007;144:654-64.

11. Mangas A, Coveñas R, Bodet D, Geffard M. Antisera and immunocytochemical techniques. In: A. Mangas, R. Coveñas, M. Geffard M (eds.) Brain molecules: from vitamins to molecules for axonal guidance. Trivandrum: Transworld Research Network; 2008. p. 1-26.

12. Mangas A, Bodet D, Duleu S, Yajeya J, Geffard M, Coveñas R. Direct visualization of retinoic acid in the rat hypothalamus: an immunohistochemical study. Neurosci Lett 2012;509:64-8.

13. Paxinos G, Watson C. The rat brain in stereotaxic coordinates. Sydney: Academic Press; 1982.

14. Mangas A, Coveñas R, Bodet D, Duleu S, Marcos P, Geffard M. Vitamins in the monkey brain: an immunocytochemical study. J Chem Neuroanat 2009;38:1-8.

15. Mangas A, Coveñas R, Geffard K, Geffard $\mathrm{M}$, Marcos P, Insausti R, et al. Folic acid in the monkey brain: an immunocytochemical study. Neurosci Lett 2004;362:258-61.

16. Alonso JR, Frotscher M. Organization of the septal region in the rat brain: a Golgi/EM study of lateral septal neurons. J Comp Neurol 1989;286:472-87.

17. Sheehan TP, Chambers RA, Russell DS. Regulation of affect by the lateral septum: implications for neuropsychiatry. Brain Res Rev 2004;46:71-117.

18. de León M, Coveñas R, Narváez JA, Tramu G, Aguirre JA, González-Barón S. Somatostatin-28 (1-12)-like immunoreactivity in the cat diencephalon. Neuropeptides 1991;19:107-17.

19. Swanson LW, Cowan WM. The connections of the septal region in the rat. J Comp Neurol 1979;186:621-56.

20. Swanson LW, Köhler C, Björklund A. The limbic region: I. The septohippocampal system. In: A. Björklund, T. Hökfelt, L.W. Swanson (eds.) Handbook of chemical neuroanatomy. 5. Amsterdam: Elsevier; 1987. p. $125-277$.

21. Gray JA, McNaughton N. The neuropsychology of anxiety. An enquiry into the function of the septo-hippocampal system. Oxford: Oxford University Press; 2000.

22. Nazliel B, Taskiran D, Irkec C, Kutay FZ, Pogum S. Serum nitric oxide metabolites in patients with multiple sclerosis. J Clin Neurosci 2002;9:530-2. 